\title{
Blood Cardiac Troponin I Increased
}

National Cancer Institute

\section{Source}

National Cancer Institute. Blood Cardiac Troponin I Increased. NCI Thesaurus. Code C78230.

A laboratory test result which indicates increased levels of cardiac troponin I in a biological specimen. 